\title{
A Semantic Memory Bank Assisted by an Embodied Conversational Agents for Mobile Devices
}

\author{
Francisco Seron ${ }^{1}$, Angel Zaldivar ${ }^{1}$, Alfonso Blesa ${ }^{2,}$,, Jose Martin-Albo ${ }^{3}$, Juan Magallon ${ }^{1}$ \\ ${ }^{1}$ Department of Informatics, University of Zaragoza, Zaragoza, Spain \\ ${ }^{2}$ Department of Electronics, University of Zaragoza, Teruel, Spain \\ ${ }^{3}$ Department of Psychology and Sociology, University of Zaragoza, Teruel, Spain
}

Email address:

seron@unizar.es (F. Seron), zaldivar@unizar.es.com (A. Zaldivar), ablesa@unizar.es (A. Blesa), jmartina@unizar.es (J. Martin-Albo), magallon@unizar.es (J. Magallon)

${ }^{*}$ Corresponding author

\section{To cite this article:}

Francisco Seron, Angel Zaldivar, Alfonso Blesa, Jose Martin-Albo, Juan Magallon. A Semantic Memory Bank Assisted by an Embodied Conversational Agents for Mobile Devices. Engineering and Applied Sciences. Vol. 6, No. 1, 2021, pp. 1-17. doi: 10.11648/j.eas.20210601.11

Received: February 1, 2021; Accepted: February 8, 2021; Published: February 23, 2021

\begin{abstract}
Alzheimer's disease is a type of dementia that causes memory loss and interferes with intellectual abilities seriously. It has no current cure and therapeutic efficiency of current medication is limited. However, there is evidence that non-pharmacological treatments could be useful to stimulate cognitive abilities. In the last few year, several studies have focused on describing and under- standing how Virtual Coaches (VC) could be key drivers for health promotion in home care settings. The use of VC gains an augmented attention in the considerations of medical innovations. In this paper, we propose an approach that exploits semantic technologies and Embodied Conversational Agents to help patients training cognitive abilities using mobile devices. In this work, semantic technologies are used to provide knowledge about the memory of a specific person, who exploits the structured data stored in a linked data repository and take advantage of the flexibility provided by ontologies to define search domains and expand the agent's capabilities. Our Memory Bank Embodied Conversational Agent (MBECA) is used to interact with the patient and ease the interaction with new devices. The framework is oriented to Alzheimer's patients, caregivers, and therapists.
\end{abstract}

Keywords: Semantic Embodied Conversational Agents, Semantic Knowledge, Alzheimer's Disease, Mobile Computing, Virtual Coaching (VC)

\section{Introduction}

Alzheimer's disease is the most common type of dementia. It is a degenerative neuropathy that causes memory loss, changes in mood and diverse cognitive problems. Alzheimer's worsens over time and, unfortunately, has no known cure. The global estimated cost of dementia in 2015 was US 818 million. Alzheimer's represents up to $80 \%$ of dementia cases.

There are two environments in the daily time in patient treatment. On the one hand, the relationship with the specialists, whatever they are (psychologist, psychiatrist, nurse, therapist, etc.). It is a complex relationship with diagnoses, treatments, prognoses, agendas, protocols, follow-ups, etc., where the healthcare system (with undoubted specialized professional value) invests a lot of resources and effort in improving the patient's quality of life.

On the other hand, the daily activity done in patients' homes, in which the figure of the main caregiver stands out: a person who is usually very close to the patient and who is completely dedicated to their care, without the resources and knowledge of professionals previously cited. In the most extreme cases, caregiver burnout syndrome (e.g. $[1,2])$ can be reached, characterized by a state of emotional exhaustion, stress and tiredness, that appears on people who dedicate much of their time to caring for a dependent person. In addition, the experience is even more difficult to manage if there are emotional aggravating factors, as happens in the 
case of Alzheimer disease.

Professionals care involves a high economic cost and is limited in time, usually a few hours a day at best. The rest of the time, caregivers take care of a patient and they bear a burden at a much lower cost.

In addition, studies point out that Alzheimer's disease and dementia $(\mathrm{AD})$ in older adults are increasing, and research effort requires new solutions to mediate this growing problem. Thus, one of these new solutions involves the use of mobile devices to stimulate the cognition of older adults and patients suffering from $\mathrm{AD}[3]$.

Currently, there is no treatment on the market able to stop the process of cognitive decline and most disease treatments are based on symptomatic medication. On the other hand, non-pharmacological treatments for dementia have reported benefits in multiple research studies [4, 5]. Especially, the popularity of Computer Cognitive Training (CCT) has been steadily increasing, as is believed to be safe and can be inexpensive [6].

Virtual coaches are seen as key drivers for health promotion in home care settings. The current evidence shows that home-based programs are effective. The term Virtual Coach (VC) refers to a coaching program or Smart device aimed at providing coaching support through a variety of applications. The virtual coach will be able to personalize and adapt goals according to the progress achieve by the user in their recovery from their impairments or disabilities. The virtual coaches guide and interaction with the user, with the aim of supporting positive actions or assisting in remembering of memory of the user. Virtual coaching (VC) is becoming increasingly important in medical care and in health-related research investments. For example, the recent EU's Horizon 2020 research framework program funded several projects on VC through a specific call entitled "Personalized coaching for well-being and care of people as the age" [7]. The primary objective of this call was the development of VC solutions that provide personalized advice, guidance, and follow-up support for key age related issues of daily life that sustain a person's abilities to remain active and independent.

The latest technological developments have shown promise as effective, accessible, and cost-effective solutions. Recent research about the use of embodied conversational agents (ECAs; a computer generated cartoon-like character, with the ability to produce and respond to verbal and non-verbal communications) has shown that user can efficaciously form a working Alliance relationship with a non-human agent (i.e., a virtual coach) [8].

$\mathrm{VC}$ and ECA are present on several disciplines: In clinical psychology there are ECA applications in the treatment of mood, anxiety, psychotic, autism spectrum, and substance use disorders [9]. There are experiences on psychotherapy and psycho-education for benefits in adherence in the screening, diagnosis, and treatment of mental illnesses [10]. ECAs are used on other psychological fields [11-13].

There are some issues related with VC and ECAs: Same works $[9,10]$ appeal to "evidence on their clinical application remains scarce". The possibilities of computer-based cognitive training (CCT) in postponing the onset of dementia are currently unclear [14], but promising [15]. The moderate effects on cognition, and anxiety and small effects on depression, could be related to the lack of recreation and cognitive stimulation of the patient himself in these intervention programs. However, the use of CCTs based on the user's own life, using their memory, may be a more attractive tool than the usual CCTs. Music [16], movies, photographs of family places and events [17, 18] and Alzheimer's have a strange and fascinating relationship. Patients in an advanced stage of the disease experience striking reactions when relating to their own memory, unlocking memories and cognitive capacities that are interesting for the patient himself.

So, one possible solution could lie in the use of the Memory Bank Embodied Conversational Agent (MBECA). The key idea of the Memory Bank Embodied Conversational Agent (MBECA) is to reproduce the memory of a patient on the cloud, in which his memory bank will be stored. The memory bank consists of a set of selected and related memories, which are supported in a variety of format such as video, music, picture, audio, text, etc. Memories can be putted by the patient, caregivers, familiars or therapists in order to boost the memories of the person. Any of the roles can use the functions of the system but each of them usually will concentrate on a determined functions set. Basically, the patient will navigate through his memories, the familiars will create and label memories, and the therapists will create/modify tags and link the memories. Human-computer intelligent interaction is an ongoing research effort that aims to provide humans with natural ways to use computers as aids. It is argued that to be able to interact with humans naturally, a computer must own the same means of communication and be able to handle knowledge, which is recognized to be a crucial part of human intelligence and natural interaction.

Some efforts in natural interaction have reported visible results. Since the introduction of Siri in 2011, spoken language understanding (SLU) [19] is present within most of the actual mobile devices as a "personal intelligent assistant" [20]. These systems can only per- form one operation at a time and still cannot follow a conversation or have initiative. Still, SLU is moving forward and new methods and applications are arising. Related to spoken language and natural interaction, Embodied Conversational Agents (ECA) [21] are graphical interfaces capable of using verbal and non-verbal modes of communication to interact with users in computer-based environments. ECAs have had a moderate widespread use (e.g. chatter-bots, automated online assistants, video games), however, its knowledge is often encoded with Artificial Intelligence Markup Language (AIML) and its scope cannot be easily changed.

The most related work to ours is the system sketched in (cite hidden for review). However, they focus on providing an extension of AIML with SPARQL to enhance the agent dialogue, while our approach, as we will see, focuses on 
exploiting the structure of data to perform searches and provide a point of access to the Web of Data using ECAs, as advocated in [22]. Although there are other approaches that use Wikipedia as its source of information for different tasks [23-25], to the best of our knowledge, our approach is the first one to exploit structured data stored in Linked Data repositories, taking advantage of the flexibility provided by ontologies to define search domains and expand the agent's capabilities.

Our focus within ECAs is to improve the quality of the information avail- able by the agent. Its knowledge must be structured and semantically labelled, providing the means to reason and react to changes in the environment. We call these improved version Semantic Embodied Conversational Agents (SECAs) (Cite hidden for review). Particular emphasis is placed on three points: the (a) appearance,

communication methods and (c) knowledge available.

1. Appearance is significantly important. An agent whose purpose is to provide medical information could be dressed as a nurse, while an agent to provide military information could be a soldier. While appearance is mainly affected by its expected application, users should also be taken into account. For example, a user with cognitive impairment may not be able to properly process a very detailed agent.

2. Communication methods are mainly affected by users and deployment plat- form. Some user groups may find natural to interact with agents using spoken language, while others might prefer to use keyboard and mouse. Also, the communication methods will depend largely on the platform where is running.

3. In order to provide the proper knowledge to the SECAs, the underlying data needs to be structured and correctly labeled. While this can be achieved using different approaches, we want our architecture to be able to manage a wide range of domains and our data model to be easily modifiable and shareable. To do so, we adopted some concepts of the Semantic Web, particularly ontologies. Ontologies, defined by Tom Gruber as the specification of a conceptualization [26, 27], allow to model and capture the semantics of different knowledge domains. By using ontologies and its related Semantic Web technologies, data models become flexible enough to that future revisions and context switch not necessarily mean big changes within the system. Thus, SECAs are able to offer a broad and deep knowledge of large domains, while still interacting in a natural way with users. Virtual characters equipped with these new features can be used in a wide range of contexts [28-30], including education and learning [30-33], sign language interpretation [34], therapy [35], persuasion [36], and entertainment [37], among others.

The goals of this work are:

1. Create an ontology that tries to provide partial support to some of the abstractions of the human memory. The proposed memory bank is a structured data store containing memories. Within this system, users are able to create, modify and explore memories and its related content. The main goal is to allow Alzheimer's patients to explore its memories as a complement to cognitive training activities. Moreover, as memories are stored in a structured way, the user can add personal information to the memory bank.

2. Build a simple and friendly interface that allows navigating by a defined knowledge universe. To illustrate our system's potential, we have built a prototype for mobile devices, concretely an Android App. The reasons for using a mobile platform to implement our prototype are as follow: semantic and web technologies are already present in Android, so no further efforts are required to migrate some solutions; patients, familiars and therapists can add and query memories and multimedia data regardless of their situation; mobile devices are highly interactive and versatile.

3. Carry out a first study of the user experiences in order to verify the functioning of the Beta version of MBECA.

The structure of the paper is as follows. Section 2 presents the conceptual premises that determine the proposal. Section 3 details the architecture of the system. Also, the semantic data model is described. Section 4 focuses on implementation details of the proposed system. Section 5 deals with the evaluation process of the framework, showing a study of the user experience. Finally, the conclusions and future work.

\section{Conceptual Premises}

The conceptual premises that support the developed system are as follows:

1. The main objective of the system is to allow Alzheimer's patients to explore its memories. This exploration is conceived of as support of the therapist intervention and is meant as a complement to CCT. Recently, a meta- analysis [49] found no evidence of the effect of CCTs when compared to active control interventions, which is expected since there are two different types of interventions with the patient. The first is a human expert, the second is usually an application of general-purpose, even if adaptable. MBECA-based CCTs introduce emotional and personal stimuli, based on the patient's memory, and can significantly modify the interaction with them.

2. Alzheimer's patients are the main focus group. Thus, interface components and navigation must be highly intuitive and humanized. Familiars and friends intended usage is to add data to the system. Their task should be improving the Memory Bank adding information as rich as possible. Finally, therapists focus into explore patient memories and could use some of its information to improve CCT activities.

3. We adopt the usage of Semantic Embodied Conversational Agents (SECAs). These kind of agents are ECAs whose knowledge is semantically enhanced using ontologies. As traditional ECAs, they interact 
with its environment through a virtual body, engaging in conversation and employing the same verbal and nonverbal means that humans do (such as gesture, facial expression, and so forth). Besides, SECAs knowledge must be semantically described, enabling further features, as they could interpret some meanings behind the actual data.

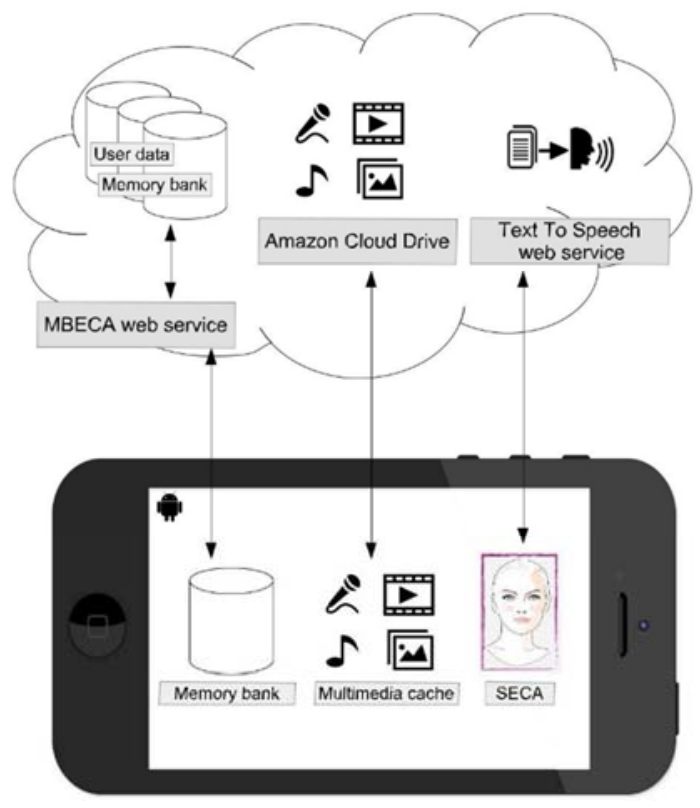

Figure 1. General architecture.

4. In order to enhance ECAs semantically, the proposed system adopts a data model focused on the use of ontologies. Ontologies permit us to represent our knowledge formally and, using technologies associated to the Semantic Web, store information in a structured manner. Improving the data model by using these technologies allow the system context to be more expressive and flexible. Moreover, as ontology models are easily expandable and exchangeable, most elements within our system could be reused to exploit information from other domains.

5. The standard for representing ontologies is the Web Ontology Language (OWL). This language is built around the Description Logics formalism, which makes it possible to reason about their defined entities using the so called DL reasoners. The actual data that follows the model described in ontologies is represented using the Resource Description Framework (RDF), a Semantic Web standard used to represent the information as a graph.

6. It's assumed that, in the future, users would talk directly to the computers and that they would carry out the commanded tasks. However, so far, there is no a perfect solution to natural language processing due to the high complexity of human language. On the other hand, the use of Graphical User Interfaces (GUI) is widespread, and it became the actual reference in human-computer interaction. Thus, we decided to adopt a hybrid communication process, using both human means and classic computer-human interaction. While user input is retrieved using classic GUI elements, both SECA's human means and GUI elements are used to show output results.

Therefore, under these premises, the restricted form of natural language that our ECAs use when dealing with a well-established knowledge domain, allows us to build interfaces that offer the users to use their natural languages, whether in a written or spoken way, while the computer detects and extracts the most significant keywords from the speech, and performs a semantic search on the underlying data repository. In the following section, we overview the architecture of our proposed approach, which is based on these presented premises.

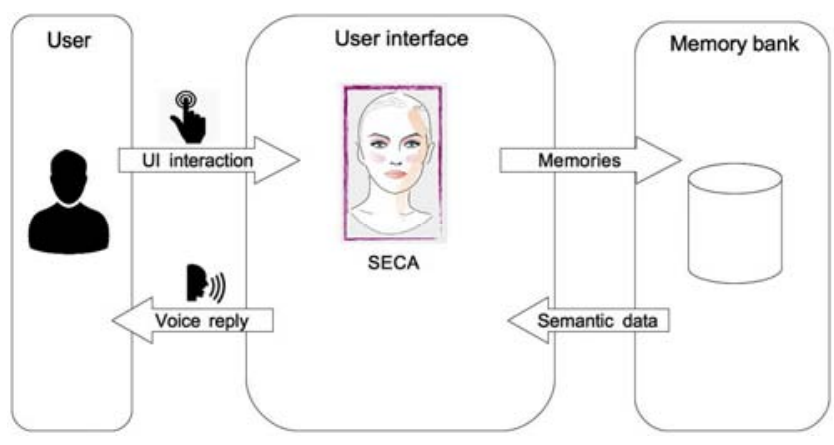

Figure 2. Interaction scheme.

\section{Proposed Framework}

The application is designed as a client-server architecture. The client side is the mobile application with the multimodal interface implemented in Android. The servers side is a set of web services that allow the access to RDF repository and the multimedia gallery. All the available user data is stored in the RDF repository into the user profile (own graph) and constitute its memory bank [38]. The data repository is synchronized with new data are detected. For the multimedia gallery, a client cache is implemented. Also, the web services allow the user authentication from the client app. The server side can be deployed in the Amazon Elastic Compute Cloud [39] for a right scalability. The server side was implemented with Restful technology using Java language. The requests use the HTTP standard (GET, POST, PUT, etc.).

Figure 1 shows the architecture point of view our system, which is com- posed of two main modules:

1. User interface based on an ECA. It is responsible for capturing all the inputs, and processing and enrouting them to the appropriate underlying services. Moreover, this module is also in charge of generating the system's reactions and the animations of the $3 \mathrm{D}$ virtual agents, providing the visual dimension of the agent as shown in figure 2 .

2. Memory Bank Module. It provides access to the semantic knowledge stored in Linked Data repositories, which are attached to the system by defining their search domain using ontologies. 


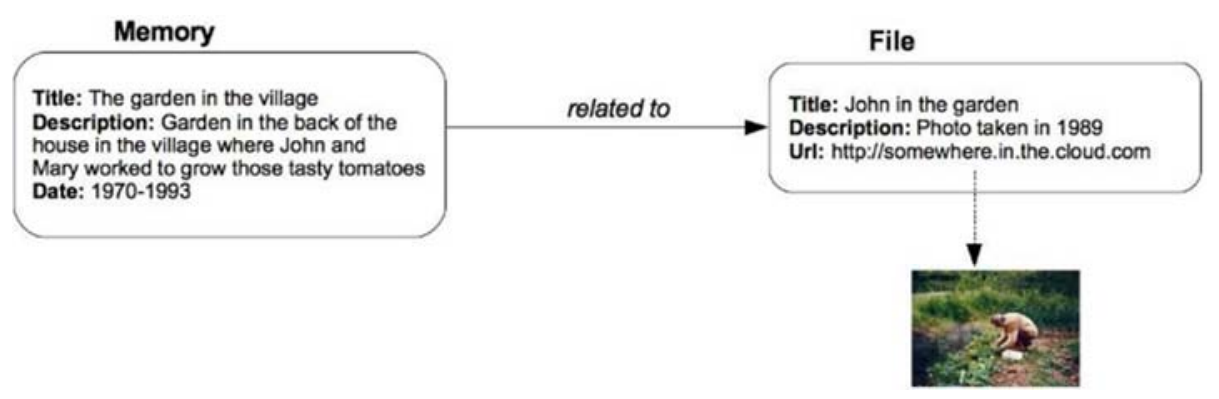

Figure 3. Memory instance.

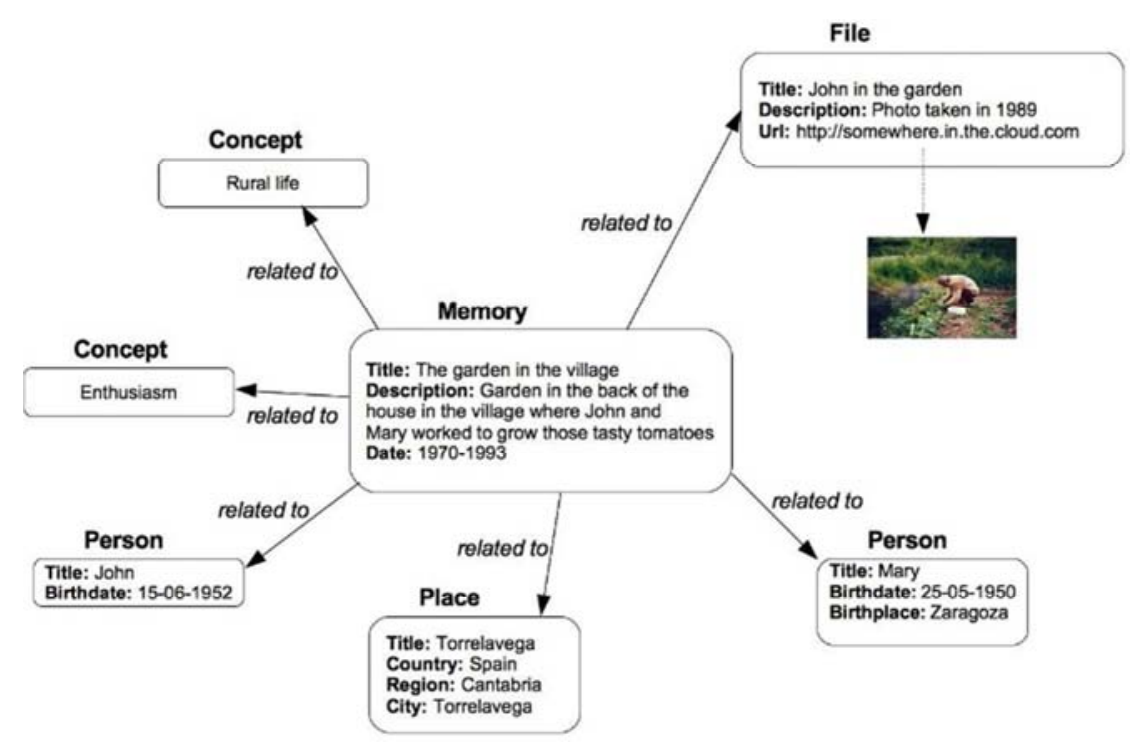

Figure 4. Memory instance.

Finally, the ontology is stored in a Virtuoso repository [40], which is an RDF repository which provides access to the stored data via an SPARQL endpoint. SPARQL is the W3C standard language for querying RDF graphs, once that we have built the appropriate queries in SPARQL, pose it to the final endpoints and access the actual data. The Jena API was used for accessing the data repositories [50]. The Domain Ontology has to be implemented in OWL, and we have used Protg [41], an ontology editor and knowledge-based framework, to edit and annotate it with the information needed to define the search domain in a proper way.

In the following subsection, we present the structure of the information accessed by our ECA and, then, in Section 4, we explain how our system exploits it.

An ontology is a formal naming and definition of the types, properties, and interrelationships of the entities that really exist in a particular domain of discourse. The model compartmentalizes the variables needed for some set of computations and establishes the relationships between them. Most ontologies describe individuals (instances), classes (concepts), attributes, and relations.

First, along with the language information detailed in the previous section, our agent has to be provided off-line with the definition of the search domain that it is going to be used. The definition of the domain is done via specifying the concept hierarchy of the domain (i.e. the classes of the objects that are considered by the agent in the searches), and annotating their properties with additional information that guides the agent in the search process. These an- notations provide the agent with information about which properties it must use in the search and return as part of the answer, and about the language that should be used when considering them. We refer the interested reader to [42] for further details on the domain definition.

According to [42], the domain definition should be specified in a single ontology. The proposed ontology has two parts. The first one contains the classes and properties needed for memory description, also contains other relevant classes closely linked to one another. These elements are defined using OWL language. The second one contains a set of schemes and concepts to make easy the labelling and classifying of the memories. In this case, the Simple Knowledge Organization System (SKOS) was used [38]. Its main objective is to enable easy publication and use of such vocabularies as linked data. Usually, the definition of a specific ontology uses entities from other ontologies to reuse existing concepts. The namespaces are used to enhance read ability of the specifications in large ontologies and to know where the source of each term. The prefixes are used to synthesize the URIs of the classes, properties, and instances. The external ontologies and prefixes used in the proposed ontology are detailed in table A.1 in appendix A.

The proposed ontology defines a set of classes. This classes 
will be instanced to create specific objects. Each class has a set of data properties to describe each instance. Also, to enrich the description of each element, the classes can be linked with object to object relations. These relations are defined as object properties. The figure A.2 in appendix A shows the set of Classes, Object properties and Data properties defined in the proposed ontology.

The concepts allow relating the instances with some abstract ideas. The concept schemes are groups of related concepts that are hierarchized. This hierarchy allows a thematic classification of the memories. Here, each individual is an instance of <concept $>$ class. The proposed ontology defines a set of concept schemes: Art, Emotion, Body and mind, Environment, Event, Healthy habit, History of life, Relationship and Social norm. The figures A.3, A.4, A.5, A.6, A.7 in appendix A show the hierarchy of concepts. Finally, the figure A.1 in appendix A shows the proposed ontology. The scheme contains all classes, data properties, and object properties.

In order to create a memory, an instance of $¡$ memory $i$ is created. Then, basic textual information about the memory is added to it. For example, An instance of $<$ memory $>$ is created named "The garden in the village" with the description "Garden in the back of the house in the village where John and Mary worked to grow those tasty tomatoes", as shown in figure 3 . Each $<$ memory $>$ can be linked with any number of instances. Each one represents only one object and it will have its own relationship with the $<$ memory $>$. For example, given the $<$ memory $>$ above, a file is created with the name "John in the garden", whose description is "Photo taken in 1989". Also, an URL of a photo of Mr. John working in the garden is attached. The file is related with the memory through the relation $<$ related to $>$.
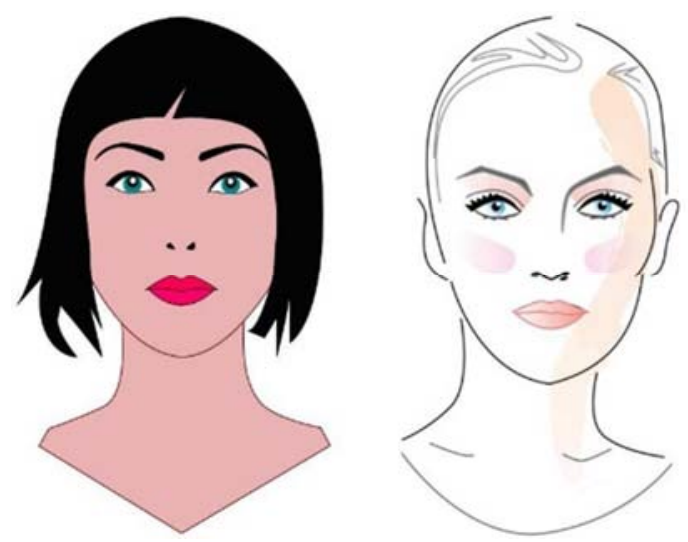

Figure 5. Left: Anthropomorphic $2 D$ agent with simple design in neutral position. Right: Anthropomorphic 2D agent with complex design in neutral position.

A $<$ memory $>$ can be linked with multiple concepts. For example, the previous example of $<$ memory $>$ can be linked with "rural life" and "enthusiasm" as shown in figure 4 . These concepts should be previously added to any of concept schemes and the relation is defined as $<$ related to $>$.
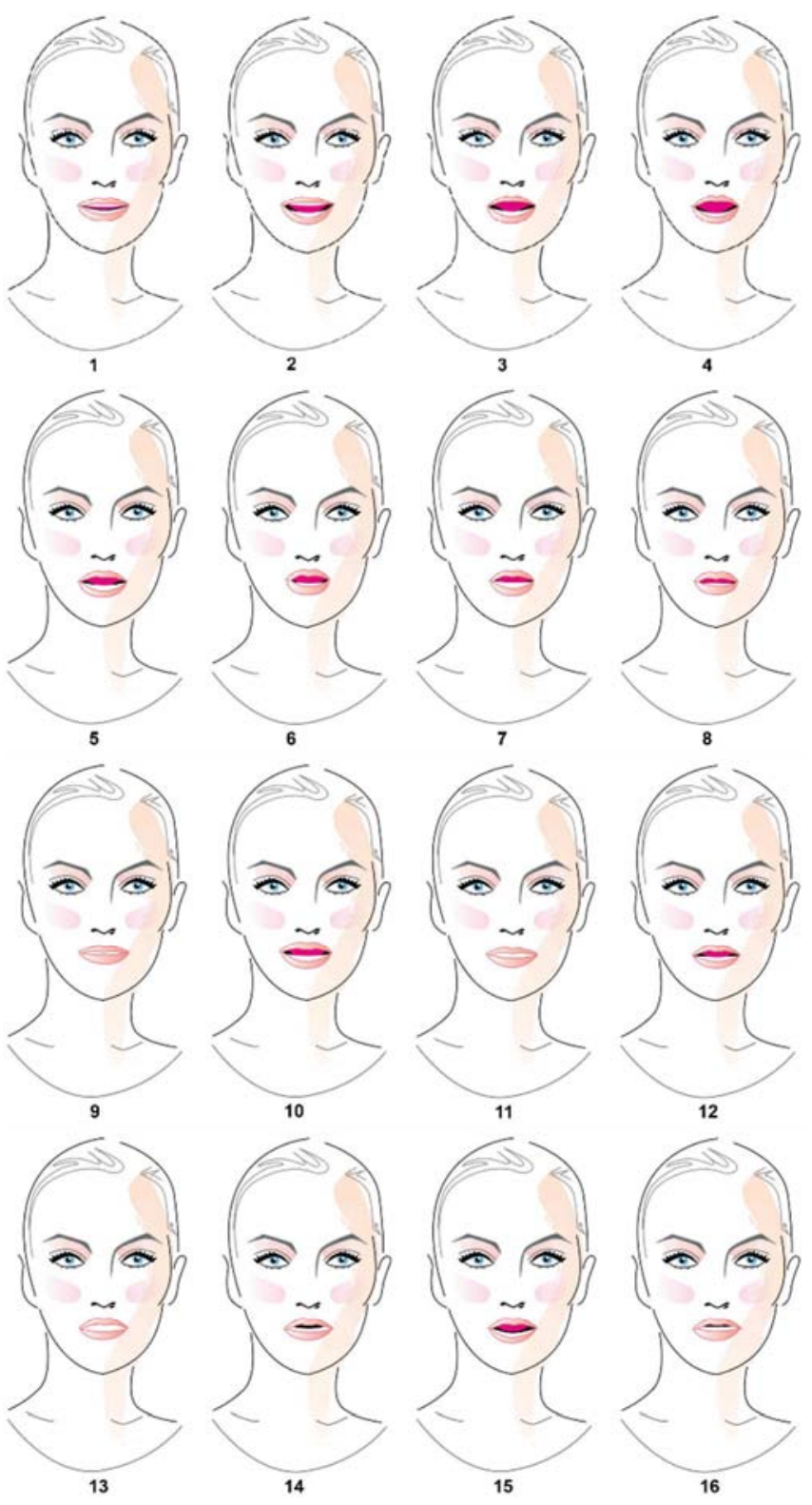

Figure 6. The 16 visemes needed for implementing the voice synthesis. Each of them is as-sociated with a set of phonemes. The viseme 1 represents the phoneme /IY/; the viseme 2 represents the phonemes /IH/, /EY/, /EH/, IAE/, $/ A H /, / A Y /, / A W /, / A N /$ and $/ H /$; the viseme 3 represents the phonemes $/ A A /$ and /AO/; the viseme 4 represents the phonemes $/ O W /$, /UW/, /AX/, /OY/ and /YU/; the viseme 5 represents the phonemes /UH/ and /ER/; the viseme 6 represents the phoneme /Y/; the viseme 7 represents the phonemes /L/, /T/ and /D/; the viseme 8 represents the phoneme $/ R /$; the viseme 9 represents the phonemes $/ M /, / P /$ and $/ B /$; the viseme 10 represents the phoneme /N/; the viseme 11 represents the phonemes $/ F /$ and $/ V /$; the viseme 12 represents the phonemes /TH/ and /DH/; the viseme 13 represents the phonemes /S/ and /Z/; the viseme 14 represents the phonemes /SH/, /ZH/ and /CH/; the viseme 15 represents the phoneme /G/; the viseme 16 represents the phonemes $/ \mathrm{J} /$

\section{Prototype Implementation}

\subsection{Visual Representation of the SECA}

One of the goals of this project is to facilitate the inclusion of SECAs in application interfaces. In next section are described the process of building of $2 \mathrm{D}$ virtual agent with 
vectorial graphics. The agent is guiding the user through auditive and visual aids while navigating in the application. Also, the agent has voice synthesis, shows a very simple set of emotions, and animation.

Due to the agent is implemented with 2D graphics using Scalable Vector Graphics (SVG) is essential that the designer takes into account certain restrictions and details [43], [44].

The methodology used for design and building of 2D agent was as follow: Taking as a basis the first version of the agent with neutral expression are created copies of the model with slight variations (e.g. closed eyes, O-shaped mouth, etc). With this procedure is achieved avoid differences among reference axis and proportions in the consecutive frames of the model. In an iterative process, the design is optimized until it is aesthetically right and functional. Figure 5 shows the two anthropomorphic agents implemented with neutral expression. The meaning of implementing two prototypes of agent is to be able to provide different levels of visual complexities according to different levels of cognitive impairment in the patient. The simplest model of the agent has a minimalist look and which is more like an animated cartoon. The empathic model of the agent has a most elaborate and artistic look. This agent can represent emotions in a most natural way.

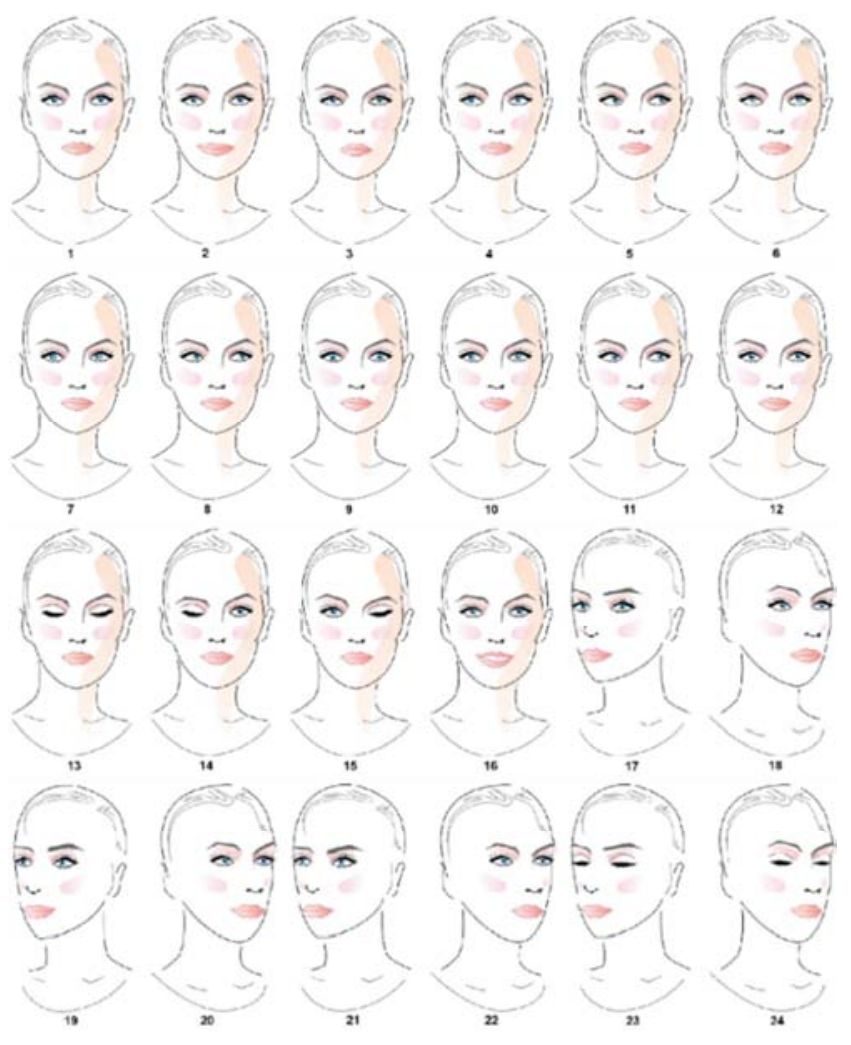

Figure 7. Empathic designs for 2D model. 1- neutral expression; 2- happy frontal expression; 3- sad frontal expression; 4- left look; 5-right look; 6- up look; 7- up left look; 8- up right loo; 9- down look; 10-down left look; 11down right look; 12-frontal look; 13-close eyes; 14- left wink; 15-right wink; 16- wide smile; 17- 3/4 left neutral; 18- 3/4 right neutral; 19- 3/4 left happy; 20-3/4 right happy; 21-3/4 left sad; 22-3/4 right sad; 23-3/4 left close eyes; 24-3/4 right close eyes.

Both models should fulfill the next requirements:

1) The format to export the agent is SVG standard.
2) The exported file should contain only useful information. The metadata relative to edition tools should be avoided for a lightweight storage.

3 ) In order to simplify the design, the model only shows the bust of the character.

4) Expressions to use: neutral, happy and sad.

5) The face is able to blink, wink the eye and show a wide smile.

6) Directions to look: up, down, left, right and front.

7) The agent is able to turn the head in $3 / 4$ (left or right). In this position can adopt any facial expression (neutral, happy or sad) or a resting posture and to blink.

8) The SECA can to talk therefore it should show a set of visemes (visual phoneme) sufficient for a correct a natural vocalization and synchronization. The model includes a set of 16 visemes. The mouth expression should be discreet to produce the effect we want. Figure 6 shows the 16 visemes. Also, the model may be placed in different kind of frames for user customization.

Figure 7 shows the stages of the agent needed for implementing the different requirements mentioned above. On the other hand, figure 8 shows the implemented frames to surround the agent.

Another requirement of the graphics model is that the different graphics entities (path, circle, rect...) are labeled with a descriptive identifier to facilitate the access from source code. For example, the entity that draws the tongue is identified with the label "tongue" instead of a number.

The model is stored in a hierarchical structure formed by groups, each of them is associated with a set of elements and that together constitute more complex forms. For example, the group identified as "mouth" is composed of the set of geometric elements "mouth background", "tongu", "upper lip", "lower lip", "upper tooths", and "upper tooths". This kind of structure facilitates the navigation by the data. Also, the hierarchical nature of the model avoids the use of absolutes coordinates and enable the relative handling of the entities.

For integrating the SVG model into the HTML5 [45, 46] document that contains the agent in the user interface is necessary to insert the SVG model in neutral expression in the body of HTML file. Here, is required adjust the viewport in order to the visible area are appropriate for the graphics size. The library Snap.svg is used to implement the animation of the vectorial model $[47,48]$.

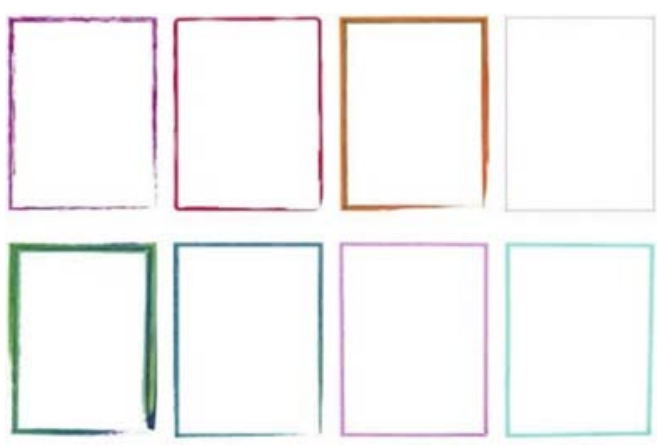

Figure 8. Frames implemented to surround the agent. 

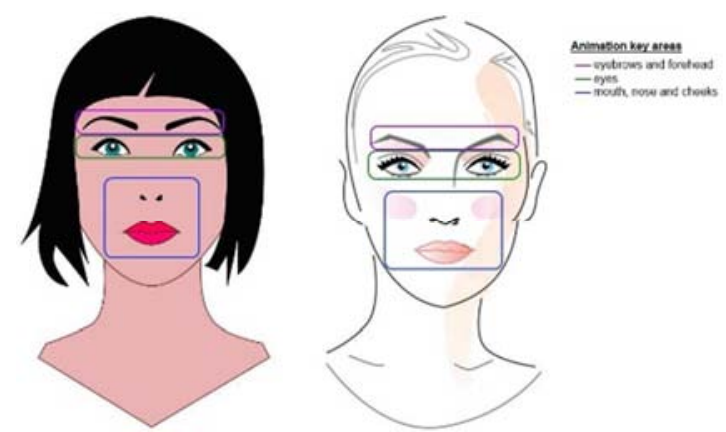

Figure 9. Key zones in the graphics area of the agent.

The animations are described by means of functions that modified the attributes of the elements involved in the animation. The function moves the attribute values to a new objective state, the functions can be chained and/or launch independently. The sequence that causes the agent to blink is composed of two functions, the first to close the eyes, and the second to back open eyes. The first function is chained with the second function, and the last one launches the first again with a pseudo-random delay. This delay simulates the natural gap between the blinks of a person. In both animations, the attributes of the eyes elements are altered, the modification of the elements are carried out simultaneously.

During the animation, the geometric elements of the model are modified. For the specific case of path elements, must be taken into consideration that the number of vertices should be inalterable in the whole sequence of images. Otherwise, the expected performance cannot be achieved. Equally importantly, the sense of direction of the path definition must be taken into account. The symmetric transformation of an element with respect an axis results in a path whose sense of direction is opposite to original element. If the new path is used in an animation, an unwanted overlapping effect can arise. The best solution to this situation is to invert the sense of direction of the new elements after the symmetric transformation.

The groups that will be animated are handled in a special way. These groups form key zones into the graphics area. In the case of 2D model are considered that the key zones are:

1) Eyebrows, this include forehead and eyebrows.

2) Eyes, this include eyelids, pupil, and iris.

3) Mouth, this include cheeks, nose, and mouth.

Figure 9 shows the different key sections of the two implementations of the anthropomorphic agent.

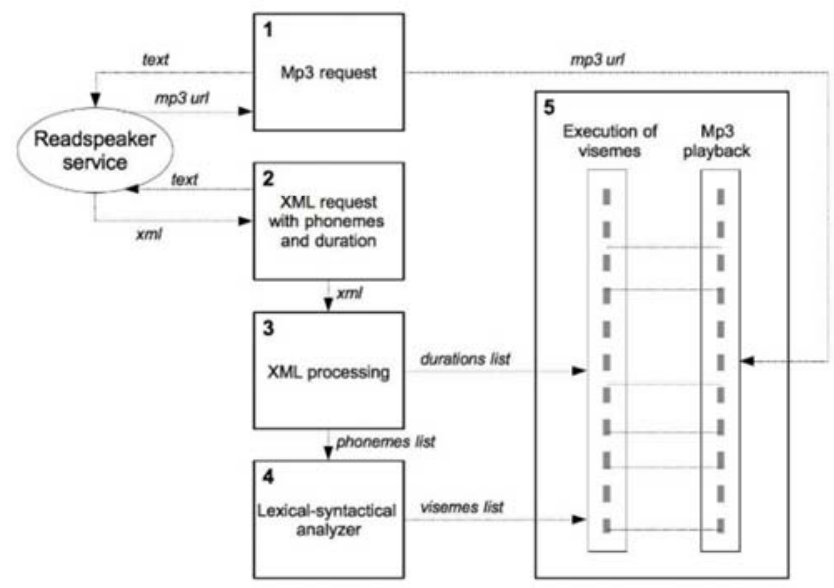

Figure 10. Steps to follow in order to synchronize the audio and the visemes during the speech of the agent.

In the description of each animation should be detailed the involved groups and elements. Also, the modification to achieve the objective state should be equally detailed (independently of the origin state). The animation descriptions together with the hierarchical structure described above be able the integration process of the animation.

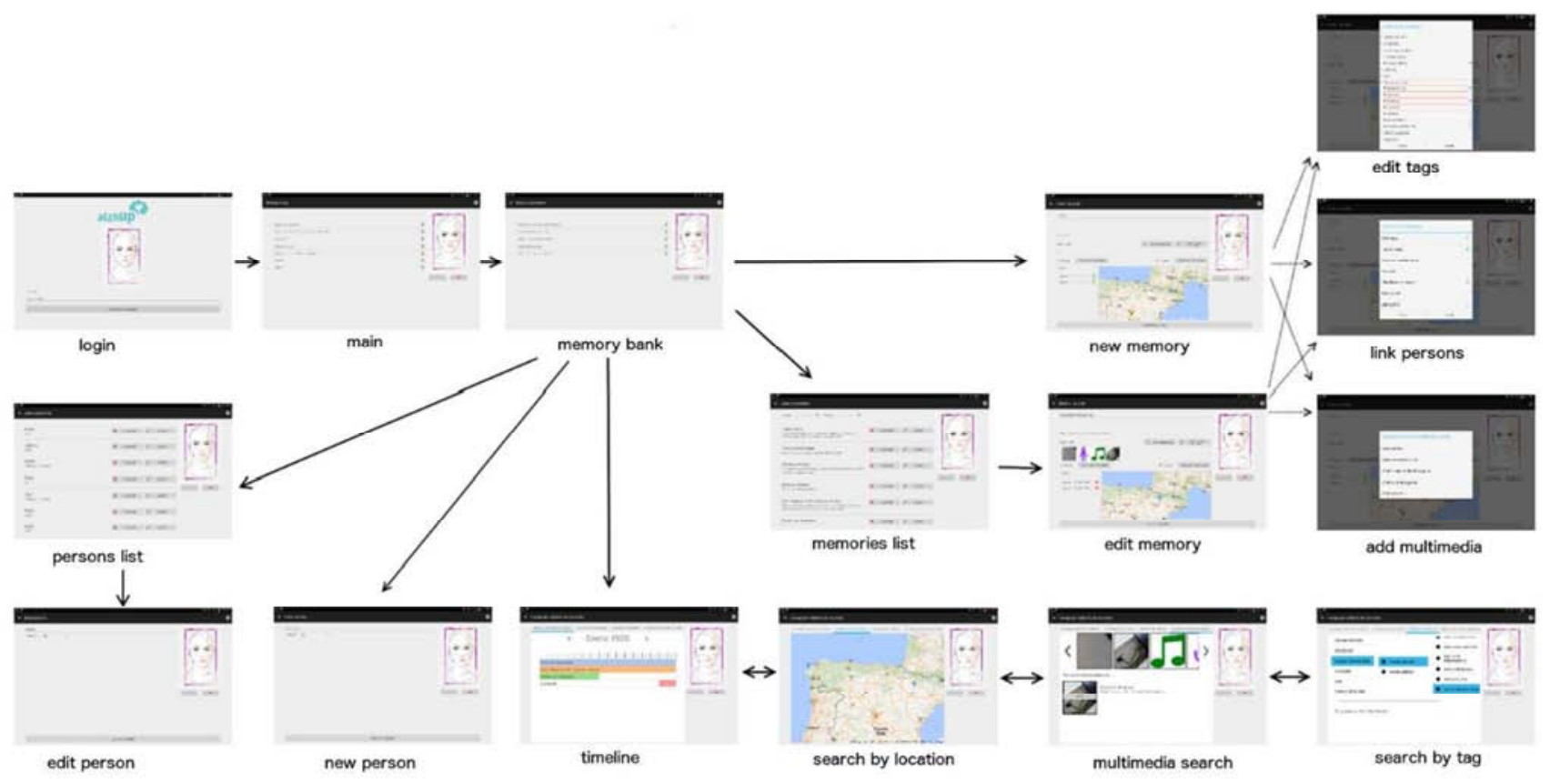

Figure 11. Navigation map. 


\subsection{Voice Synthesis}

In order to provide the agent with voice synthesis was used an online payment service of Text To Speech (TTS). Given a text string, the service returns the voice synthesis of the text, which is played by the application. In addition to the audio, the service returns the phonemes sequence that form the text and the duration of them. This extra information allows the perfect synchronization between the audio and the visemes. Figure 10 shows the process of synchronization, which perform the following steps:

1) Given the text to speech, the application requests the mp3 audio file to the service.
2) If the result is correct, the application requests once again with other parameters an xml file that contains the phonemes sequence that forms the text and the duration of them.

3) The XML file is parsed to fill the data structure in memory.

4) Then the phonemes list is passed by a lexical-syntactical analyzer to obtain the list of visemes.

5) The process ends with the reproduction of the audio synchronized with the reproduction of the visemes determined by the duration of them.

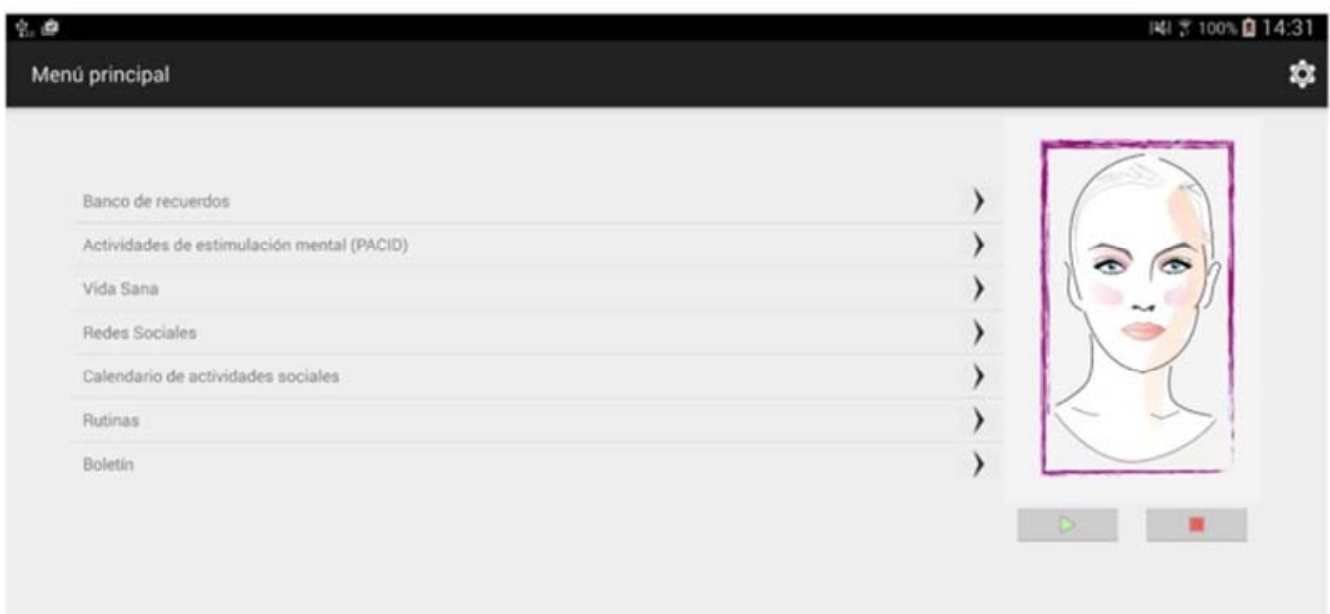

Figure 12. Menu' principal /Main menu. Items: Banco de recuerdos/Memory bank, Actividades de estimulaci'on mental (PACID)/Mental stimulation activities (PACID), Vida sana/Healthy lifestyle, Redes sociales/Social networks, Calendario de actividades so- ciales/Calendar of social activities, Rutinas/Routines, Bolet'in/Bulletin

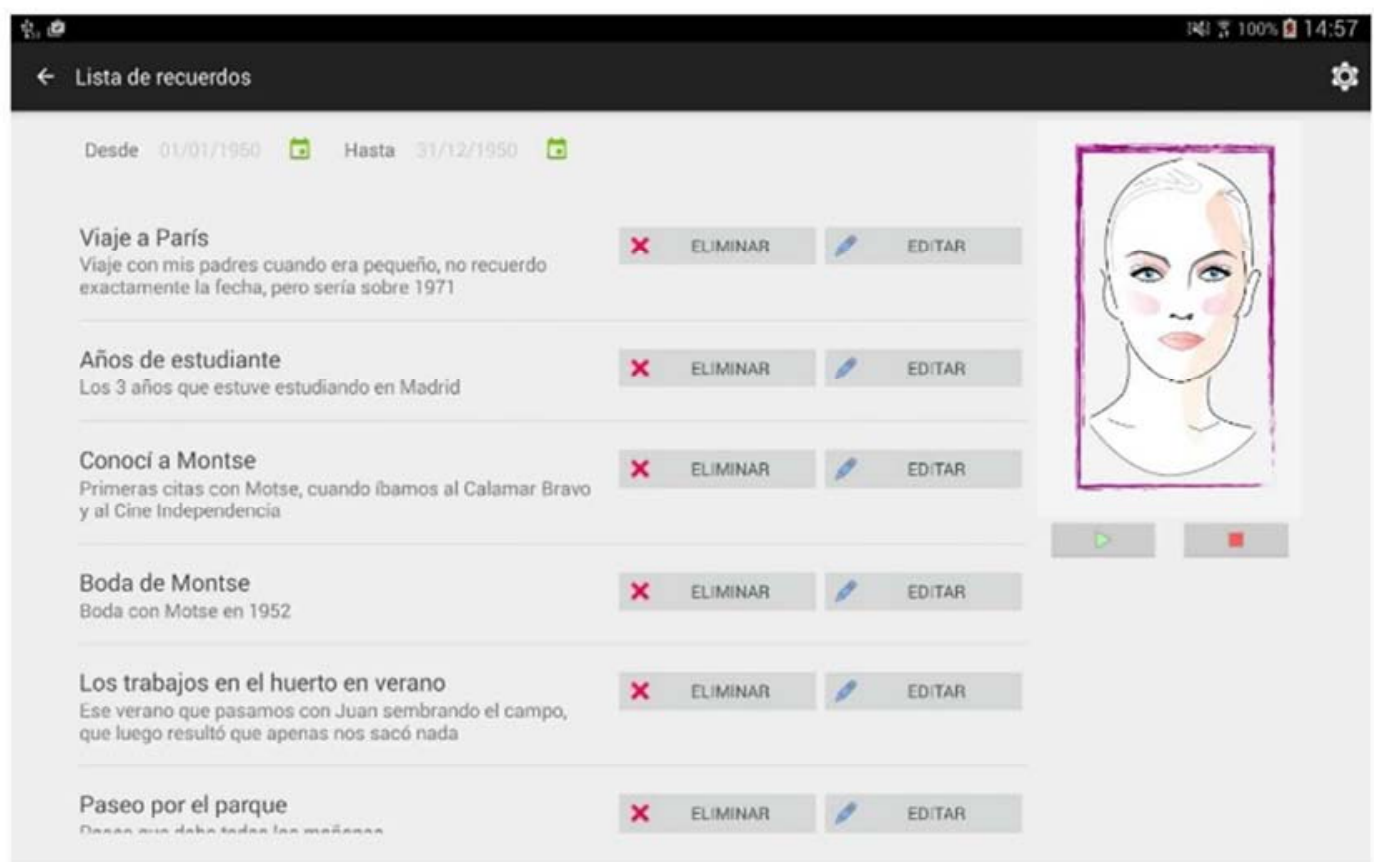

Figure 13. Lista de recuerdos/List of memories. Date filter: desde/from, hasta/to. Items: Viaje a Paris/Trip to Paris, Años de estudiante/Years of student, Conoci a Montse/I met Montse, Boda de Montse/Wedding with Montse, Los trabajos en el huerto en verano/Work in the garden in summer, Paseo en el parque/Walk in the park. Options: eliminar /remove, editar ledit. 


\subsection{Multimodal Interface}

In order to satisfy the expectations of the user and ensure a good experience in the mobile environment, the application should be adapted to standard of interaction in touch device. Some of the guidelines taken into account are:

1) Structure of information should be simple and readable.

2) Use of basic elements in touch interfaces as sliders, check boxes, tabs, text boxes.

3) Navigation based on lists.

4) Back navigation is enabled in all views. If the virtual keyboard is shown, the back button firstly closes the keyboard. Similar behavior is showed with an open pop up.

5) Depending on the needs of each view, the interface should automatically adjust to screen orientation (landscape or portrait)

6) The transitions between views can include animations.

In the proposed interface also have been taken into consideration some other design principles:

1) Due to the characteristics of potential users of the application, the simplicity should be a key principle both in interaction and navigation scheme and the interface design.

2) Each view has a virtual agent with voice synthesis, the agent can be enabled or disabled at any time. This agent guides the user during the interaction. Furthermore, a set of contextual predefined phrases are available by the agent for times without interaction.

3) The first time that the user logs in the application, its credentials are stored locally.

4) Internet connection is required all the times.

Table 1. The measure of adherence is the number of sessions and total hours of duration for each session (maxsessions=9) and (maxtotalhours=10). $N=117$ questionnaires included in this study. 2/3 days a week, for 3 weeks doing in each session a round of activities.

\begin{tabular}{lll}
\hline Hours & Questionnaires & \% \\
\hline$[0,00-2,50)$ & 48 & 49,6 \\
{$[2,50-5,00)$} & 26 & 22,2 \\
{$[5,00-7,50)$} & 18 & 15,4 \\
{$[7,50-10,0]$} & 15 & 12,8 \\
Sessions & & \\
{$[0-2)$} & 43 & 36,7 \\
{$[2-4)$} & 27 & 23,1 \\
{$[4-6)$} & 22 & 18,8 \\
{$[6-9]$} & 25 & 21,4 \\
\hline
\end{tabular}

Figure 11 shows the navigation map of the application. The next figures show the visual aspect of the prototype system that has been designed and implemented. Figure 12 shows the main menu of the application, the option "Bank of memory" can be directly accessed from here. This menu contains different options to navigate the bank of memory with different approaches. Also, the menu includes the options "Add new memory" and "Edit memory". This last option takes you to the list of existing memories to select the element that you want to modify as shown in figure 13 .
Figure 14 shows the view to introduce a new memory. The form contains all the fields that a memory may have associated. This includes basic data as title, comment, place, and date and also contains semantic relations among existing memories, types, tags or persons. The button "Add/remove tags" shows a popup that allows selecting the tags associated with the memory as shown in figure 15 . The items of the tree are taken from the described hierarchy in the ontology. As previously noted, the application allows different kinds of navigation to the memory bank. The timeline establishes a temporal scheme of the memory.

Here, a time window can be specified in the interface to filter memories in a date range. The user can navigate month to month pulsing the corresponding buttons. The tab to navigate by localization shows a map with pins to highlight the places where some memories have occurred. Another tab allows navigating through the multimedia resources of the memory bank. Also, this view contains a date filter. Figure 16 shows the tab to navigate by semantic labeling. This view allows filtering the memories by tags. The main view shows the first level of tags, when one is pressed in the top appears the subtags of the selected tag and in the bottom are shown the memories that have associated this tag. All tabs to navigate the memory bank have a high interconnection among them, pulsing any element that is represented in another tab the interface establishes a hyperlink to this one

\section{A first Study of the User Experience}

The study done by us tries to verify the adaptation and effective functioning of the Beta version of MBECA development. In this way we ponder a first stage of study mainly focused on the user experience (UX), design verification and both subjective and objective facets about adaptation device person implied on the whole social ecosystem on Alzheimer's Disease.

User-device adaptation and user experience test will be made on different study groups with a determined target audience.

Objectives: The general objectives are to determine the user experience and satisfaction handling MBECA on different target audiences. Otherwise, the specific objectives are the following:

1) To obtain objective rates of users' usage.

2) To know the subjective and emotional impressions of the user while using MBECA.

3) To verify the aspects of the UX in which graphic and environment design affect on the usage of MBECA.

4) To gather the impression (via objective methods) of the professionals about the use and user adaptation of MBECA.

5) To obtain the first execution indexes from users during therapeutic activities

Methodology: The testing and observation sessions of MBECA have been done individually, with the presence, 
depending on the case, of a professional and/or a family member. The tests have been done in agreement with the institutions in direct contact with the users.

We contacted Institutions principals to explain the aim of the research and requesting their permission to conduct this study. Next, depending on the case, passive consent was obtained from parents or guardians. All participants (parents, guardians and patients) were invited to participate and assured that the survey was confidential and voluntary.

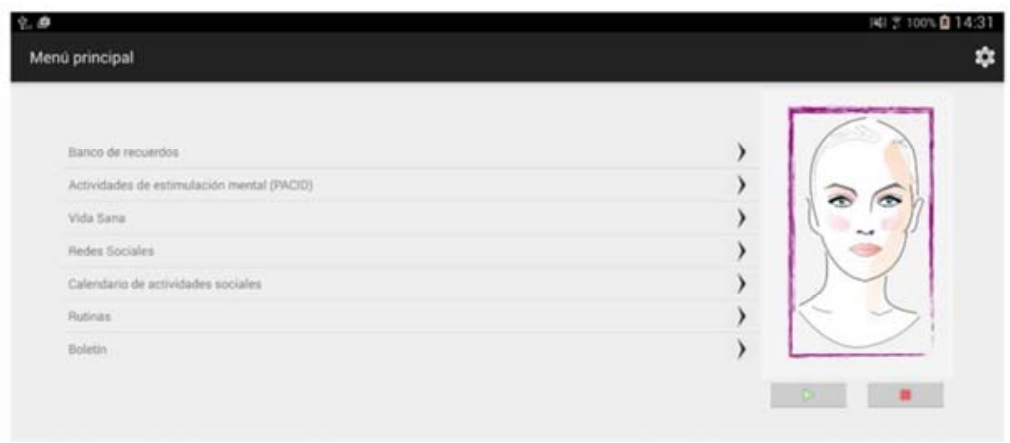

Figure 14. Title: Nuevo recuerdo/New memory. Fields: Titulo/Title, Comentarios/Comments, Multimedia/Multimedia (Options: Añadir multimedia/Add media, Listado-Eliminar multimedia/List-Remove media), Ontología/Ontology (Option: Añadir-Quitar etiqueta/Add-Remove tags), Personas/Persons (Option: Añadir-Quitar per sona/Add-Remove person), Date (fields: desde/from, hasta/to), Location. Options: Guardar recuerdo/Save memory.

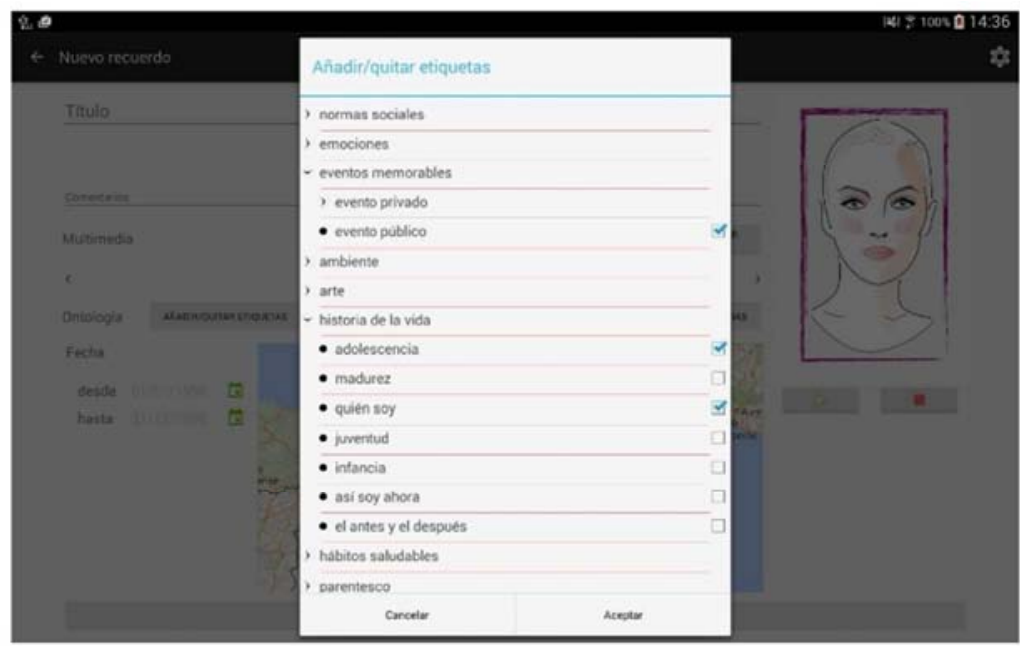

Figure 15. An adir-quitar etiquetas/Add-remove tags. Tree items: Norma sociales/Social norms, Emociones/Emotions, Eventos memorables/Memorable events, Evento privado/Private event, Evento pu'blico/Public event, Ambiente/Ambient, Arte/Art, Historia de la vida/Life story, Adolescencia/Adolescence, Madurez/Maturity, Qui'en soy/Who I am, Juventud/Youth, Infancia/Childhood, As'l soy ahora/That's who I am, El antes y el despu'es/Before and after, H'abitos saludables/Healthy habits, Parentesco/Relationship. Options: Cancelar/Cancel, Aceptar/Accept.

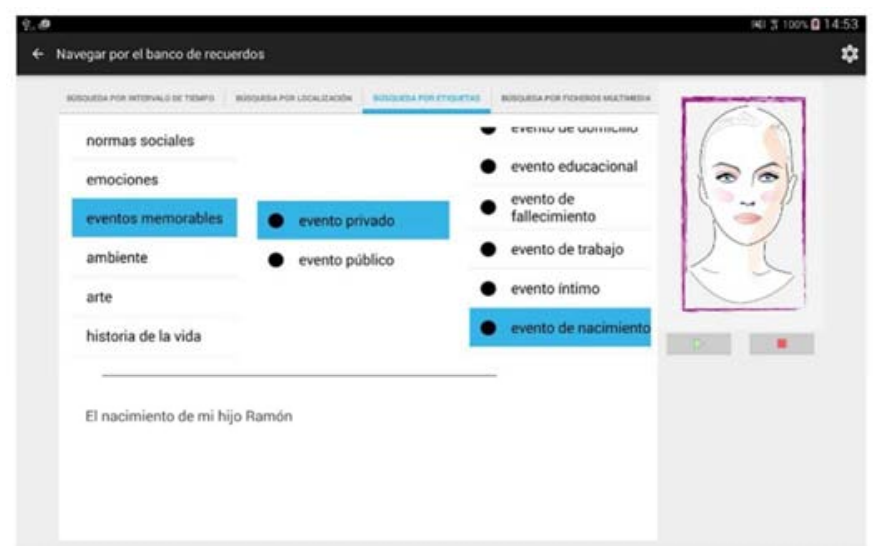

Figure 16. Title: Navegar por el banco de recuerdos/Navigate through the memory bank. Tabs: Búsqueda por intervalo de tiempo/Search by date interval, Búsqueda por localización/Search by location, Búsqueda por etiqueta/Search by tags, Búsqueda por ficheros multimedia/Search of multimedia. Selected items: Eventos memorables/Memorable events, Evento privado/Private event, Evento de nacimiento/Birth event. Result: El nacimiento de mi hijo Ramón/The birth of my child Ramon. 
Participants were chosen within an exclusion and inclusion criteria specified by the institutions. Normative data and variable control such as the experience with TIC devices, the cognitive deterioration level of the person with dementia, the training of the professionals

In the instructions how to manage the tests done the recommended protocol was to use MBECA during 2/3 days a week, for three weeks doing in each session a round of activities. Subsequently, both the person with dementia and the professional/family member should answer the data questionnaire about the experience.

Results Analysis: We have three kinds of data that are susceptible of analysis. The tests are currently on going and confirmation of this preliminary data gathering is expected. The analyzed data comes from:

1) Questionnaire from the person with dementia: questionnaire in which the opinion and UX index of the person is gathered after going through the activities with MBECA.

2) Questionnaire from the professional: questionnaire that gathers the opinion and UX index of the professional after doing the assistance work during MBECA sessions.

General data (usability and user experience). Questionnaire from the person with dementia. There have been gathered 117 questionnaires with diverse questions, each one the range goes from 0 to 5 . The descriptive results of the variables gathered by the questionnaires are shown in figure 17.

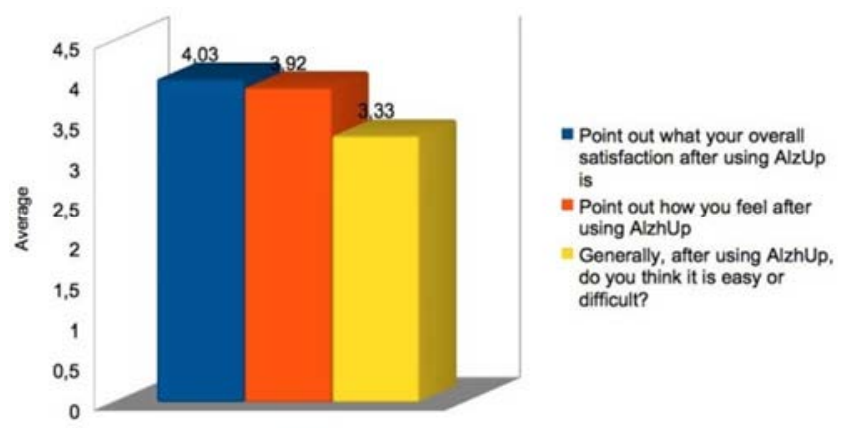

Figure 17. General data (usability and user experience). Questionnaire from the person with dementia.

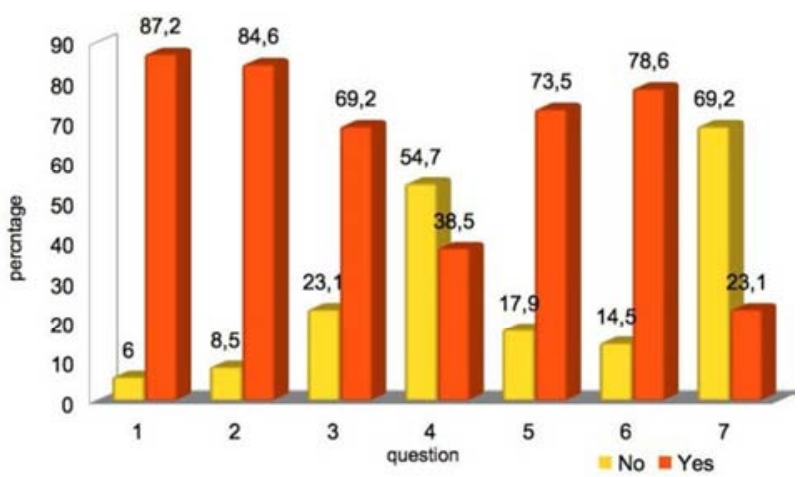

Figure 18. User experience.

The questions regarding user experience are as follow:

1. Have you understood the menus and the texts of the activities carried out?

2. Have you easily distinguished and understood the figures, memes and drawings appeared to guide you in activities?

3. 3Does the size of text and drawings, figures, etc., has been large enough to perceive and understand?

4. Have you had trouble handling the keyboard when typing, drag function or touches selection, etc.?

5. Does playing videos or sounds has been correct for your understanding and tasks?

6. Did you understand the menu selections and changes between activities, always knowing what kind of activity was?

7. Could you use MBECA independently without support from a person (caregiver, family, professional, or other)?

The results of the questionnaire are shown in figure 18 .

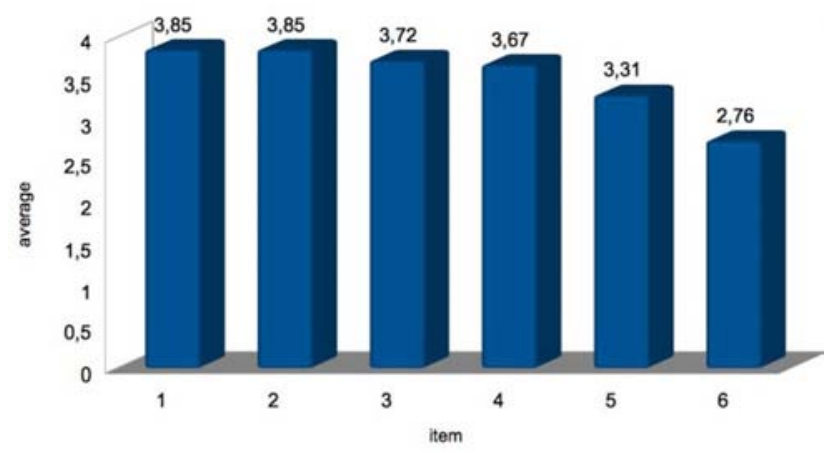

Figure 19. General data (usability and user experience). Professional questionnaire.

General data (usability and user experience). Professional questionnaire: In this case 100 questionnaires have been gathered with the implemented tests. The descriptive results from the variables gathered by the questionnaires are the following:

1. Point out what your overall satisfaction after using MBECA is.

2. Perception of the satisfaction of the person with dementia.

3. Point out how you feel now.

4. Perception of emotionality of the person with dementia.

5. Generally, do you think using MBECA is easy or difficult

6. Perceived difficulty of the person with dementia handling MBECA.

The results of the questionnaire are shown in figure 19.

Also, derived from the Professional questionnaire. About the following binary questions (Yes/No) is possible to show the following results (The results of the questionnaire are shown in figure 17.).

1. Have you understood the goal of all tasks and activities that have been made?

2. Do you think this type of activities and methodology can be therapeutic and effective?

3. Do you need some kind of support, training or advice to use MBECA therapeutically?

4. Have you understood the menus and the texts of the 
activities carried out?

5. Have you easily distinguished and understood the figures, memes and drawings appeared to guide you in activities?

6. Does the size of the text and drawings, figures, etc., has been large enough to perceive and understand?

7. Have you had trouble handling the keyboard when typing, drag function or touches selection etc.?

8. Does playing videos or sounds has been correct for your understanding and tasks?

9. Did you understand the menu selections and changes between activities, always knowing what kind of activities?

About other questionnaire of evaluation of participation and sentiment, the data is shown in figure 20. Although there is a percentage of rejection that shouldn't be scoffed at most of them don't reject MBECA. It's understating that during this first try those rejection symptoms can appear because of the novelty of the format. Regarding their level of attention and attitude shown by the users, in opinion of the professionals, from their position of participant observers, the index are as follows:

1. Indicate the general level of attention shown in the session.

2. Point the highest level of attention shown in the session.

3. Point out that attitude generally shown by the person in the session.

4. Point the highest level of attitude shown by the person in the session.

The average values of each index is shown in figure 20. Regarding the assessment of the Observed Emotion Rating Scale, the average scores are shown in the figure 21.

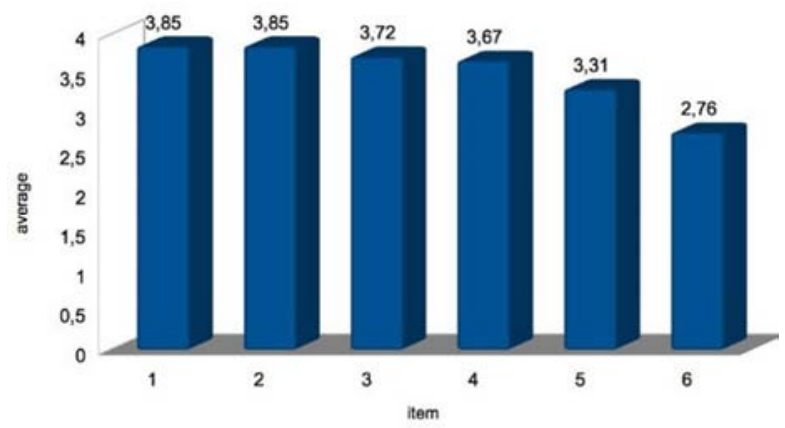

Figure 20. Level of attention and attitude shown by the users.

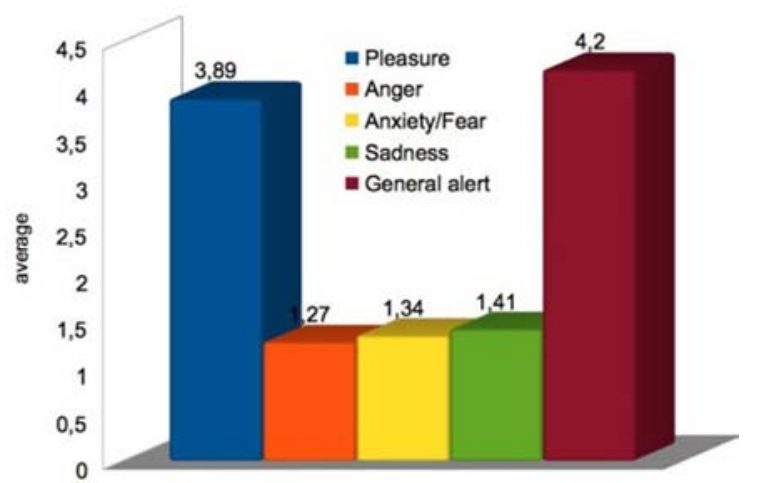

Figure 21. Assessment of the Observed Emotion Rating Scale.

\section{Conclusions}

From the point of view of a person with dementia, both the satisfaction and the emotional response of the person that uses MBECA are positive. Additionally, the interaction with the usability elements of MBECA also seems to be good on their opinion, even having a very positive perception towards self-sufficient use of the service, at least to most of them. Openly speaking, they also have said to encounter some difficulties with the "touch" functions, some "drag" and the fonts and background distinctions; those are the main complaints. Additionally, they also stand out technical issues with video playback and the speed of messages, support screens. They value MBECA very favorably and consider it novel, active at every level (cognitive and physical) and they praise their interaction possibilities with the device.

From a professional point of view, the level of satisfaction is slightly lower, but it's still positive and generates the same positive emotional response. As regards to the usability elements it's also generally positive, featuring the level of difficulty (lower score).

Additionally, they perceive it's more difficult to handle the device than the users themselves. They also state that, generally speaking, usability elements such as texts, objects and menus are readable. As we have commented before, there's a bigger proportion of people that pick up errors during video play- back as well as difficulty handling some menus. Even so, the valuation is very positive on use, and facets like image quality, ease and speed of the app have been praised, as well as support methodology and the general structure, as well as the customization. They also comment that it draws attention to the user and it's easy to use. Regarding the valuation of their involvement during the sessions, they explain that both the attitude and the attention levels are positive. And the revealed emotions, in an objective form, are positive, bringing the negative emotions during sessions to the minimal amount.

Taking all of this into account, we can specify that for both main protagonists of MBECA and the persons it's directed towards, the general satisfaction during its usage is positive. It also generates positive emotion and activates them.

It's true that it's very soon to draw conclusions, and it's not the main goal of this study, about the specific execution on each one of the different areas and activities and their relation with the cognitive decline and its evolution.

This paper presents an approach that exploits semantic technologies and Embodied Conversational Agents to help Alzheimer's patients to train cognitive abilities using mobile devices. This work continues to deepen in the interaction between ECAs and semantic technologies to power the user's experience within a controlled domain. Our SECA takes knowledge from a well-defined domain that represents the memory of a specific person captured in form of ontology. The data model formally represents knowledge about the set of concepts within that domain and the relationships that exist between them in that context. This way, the agent can focus its search on the contextual meaning of terms as they appear in 
the data space. This reduces the user's efforts to obtain their desired information and the overall amount of time spent searching. The approach adopted to define the knowledge of the agent and exploit the information sources has allowed us to develop a decoupled solution, which provides sheer flexibility when it comes to adapting our system to new scenarios.

From the point of view of user interaction, the proposed framework is focused on a set of target users that require special conditions in the interaction process. So, the work attaches special interest to the role and impact of different communication elements such as greetings, farewells, comments, sup- porting sentences, questions, intonations, and everything that makes human communication friendly.

This project outlines some methodological guidelines for the development of such complex embodied agents including integration with information systems that require its backend. With our contribution, we hope to widen the repertoire of systems that use this kind of agents, bringing them to a wider community, and to inspire them to achieve more creative and useful information systems.

A completely functional prototype has been developed, on which we have carried out an evaluation of our approach whose results are encouraging and show its usefulness. Also, the mix of development technologies shown the feasibility of our approach. However, we are aware that there is still plenty of work to be done.

In conclusion, the assessment is generally positive. There is a diversity of opinion, which on one hand points out to the diversity of all the participant actors of the research and the freedom they had when asked for their opinion; but on the other hand concerning the research of the effect of MBECA on users and their context also shows the need to delve deeper and have a bigger and more diverse sample to have more conclusive, reliable, valid results. And, of course, on a technical, functional and design level, important measures have to be taken for MBECA to be a therapeutic, useful tool for all the people involved on these kind of dementias.

The continuous technological progress is changing the $\mathrm{VC}$ impact on users. Apparent figure and partial substitute for a health professional. VC has a concrete potential, providing a great opportunity to guarantee the continuity of care between hospital and home.

So, this work develops a MBECA tool from a technical point of view, verifying that it is accepted by the potentials users it is intended for. MBECA offers benefits to patients and caregivers, being able to manage care time in a more effective way.

It remains for further work to apply it in the appropriate clinical environment, carrying out field tests and evaluating the effectiveness of MBECA in realistic situations [49]. We are considering to expand our MBECA to others areas that require special user interaction. Such as, other degenerative diseases, children with special needs and many others scenario with communication handicap. We want to remark the usefulness of this kind of agents as software components in dedicated applications domains such as public environments, homes, education, games, tele-assistance tutoring, and other commodity products from different sectors in the real world.

\section{Appendix}

Semantic data model. Figures and graphics

Table 2. Ontologies and prefixes used in the semantic model.

\begin{tabular}{ll}
\hline Type & count \\
\hline Axiom & 1253 \\
Logical axiom count & 632 \\
Class count & 7 \\
Object property count & 9 \\
Data property count & 9 \\
Individual count & 184 \\
\hline
\end{tabular}

Table 3. Ontologies and prefixes used in the semantic model.

\begin{tabular}{|c|c|c|}
\hline \multicolumn{2}{|l|}{ Ontology } & Prefix \\
\hline \multicolumn{2}{|c|}{ DUBLIN CORE METADA TERMS } & Dcterms \\
\hline \multicolumn{2}{|c|}{ RDF SCHEMA } & Rdfs \\
\hline \multicolumn{2}{|l|}{ SCHEMA.ORG } & S \\
\hline \multicolumn{2}{|l|}{ SKOS } & Skos \\
\hline \multicolumn{2}{|l|}{ XML SCHEMA } & $\operatorname{lmo}$ \\
\hline $\begin{array}{l}\text { Thing } \\
\text { Concept } \\
\text { ConceptScheme } \\
\text { Cifle } \\
\text { Oplace } \\
\text { person } \\
\text { memory }\end{array}$ & 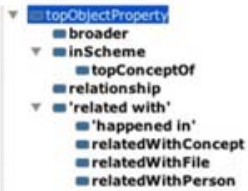 & 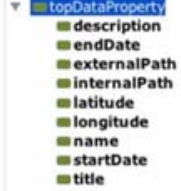 \\
\hline
\end{tabular}

Figure 22. Classes, Object property and Data property.

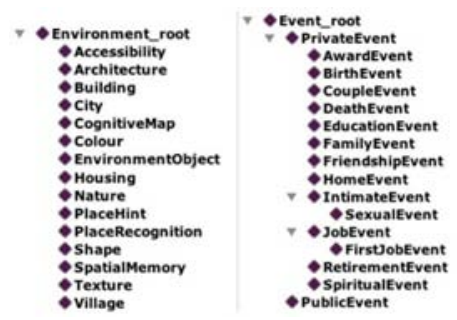

Figure 23. Instance tree of the categories Environment and Event.

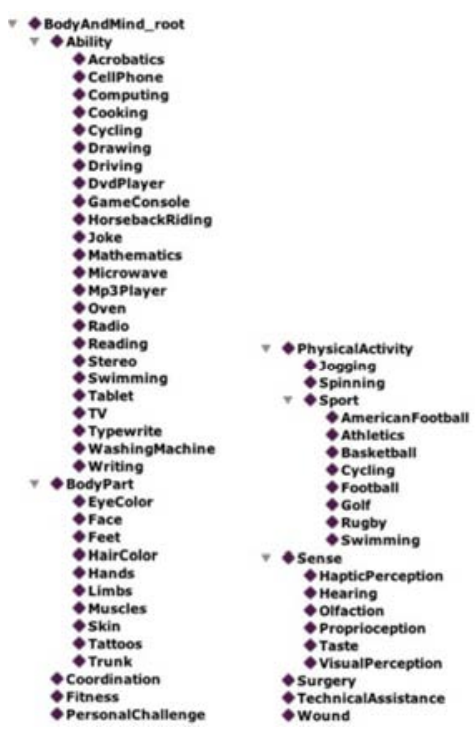

Figure 24. Instance tree of the category Body and mind. 


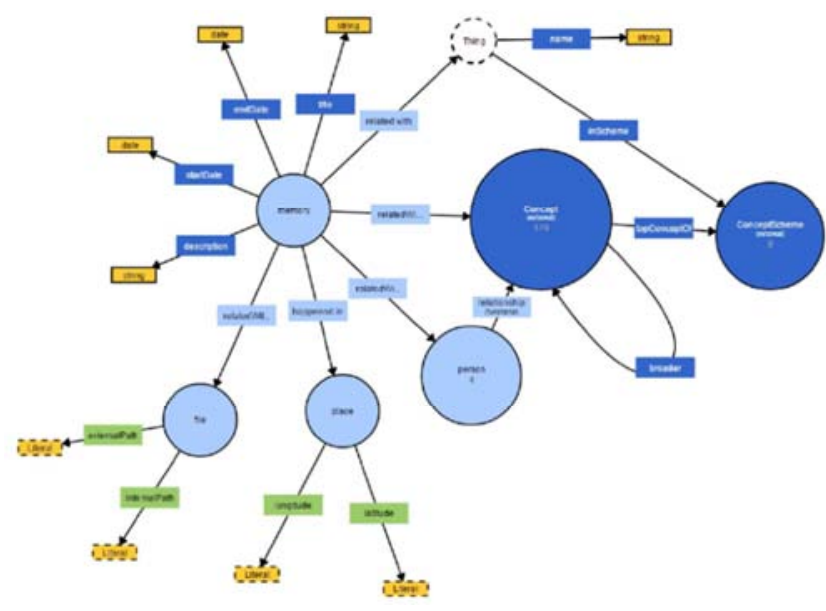

Figure 25. Ontology diagram.

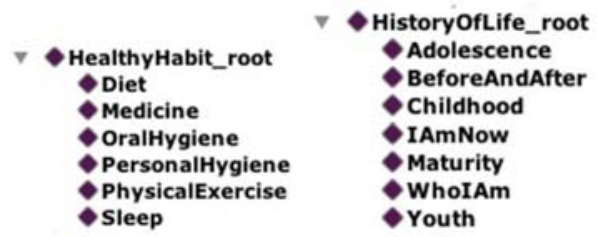

Figure 26. Instance tree of the categories Healthy habit and History of life.

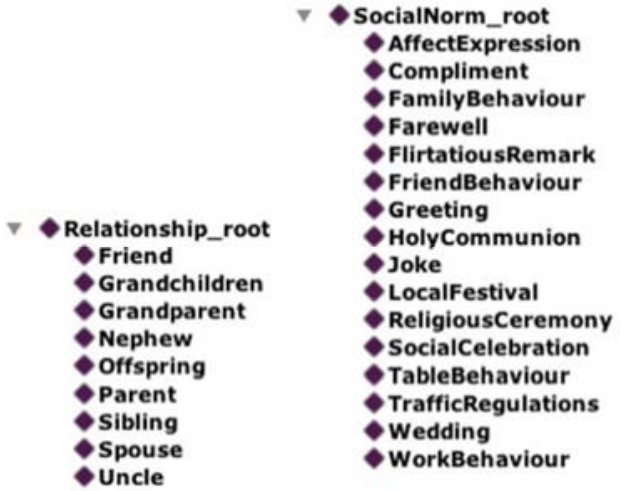

Figure 27. Instance tree of the categories Relationship and Social norm.

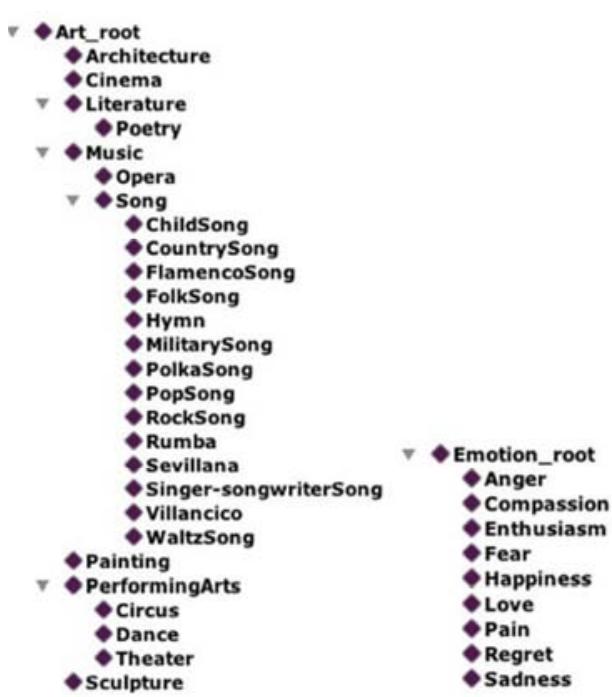

Figure 28. Instance tree of the categories Art and Emotion.

\section{References}

[1] S. Llanque, L. Savage, N. Rosenburg, and M. Caserta, "Concept Analysis: Alzheimer's Caregiver Stress," Nurs. Forum, vol. 51, no. 1, pp. 21-31, Jan. 2016.

[2] M. S. Mittelman, D. L. Roth, O. J. Clay, and W. E. Haley, "Preserving health of alzheimer caregivers: Impact of a spouse caregiver intervention," Am. J. Geriatr. Psychiatry, vol. 15, no. 9, pp. 780-789, 2007.

[3] L. Tárraga et al., "A randomised pilot study to assess the efficacy of an interactive, multimedia tool of cognitive stimulation in Alzheimer's disease," J. Neurol. Neurosurg. Psychiatry, vol. 77, no. 10, pp. 1116-1121, Oct. 2006.

[4] M. Takeda, T. Tanaka, M. Okochi, and H. Kazui, "Non-pharmacological intervention to dementia patients," Psychiatry Clin. Neurosci., vol. 66, pp. 1-7, 2012.

[5] S. Douglas, I. James, and C. Ballard, "Non-pharmacological interventions in dementia," Advances in Psychiatric Treatment, vol. 10, no. 3. pp. 171-179, May-2004.

[6] A. Lampit, H. Hallock, and M. Valenzuela, "Computerized Cognitive Training in Cognitively Healthy Older Adults: A Systematic Review and Meta-Analysis of Effect Modifiers," PLoS Med., vol. 11, no. 11, p. e1001756, Nov. 2014.

[7] European Innovation Partnership, "Personalised coaching for wellbeing and care of people as they age," European Commission. [Online]. Available: https://ec.europa.eu/eip/ageing/funding/horizon-2020/personal ised-coaching-wellbeing-and-care-people-they-age-sc1-pm-15 -2017_en. [Accessed: 03-Apr-2020].

[8] T. Bickmore, A. Gruber, and R. Picard, "Establishing the computer-patient working alliance in automated health behavior change interventions," Patient Educ. Couns., vol. 59, no. 1, pp. 21-30, Oct. 2005.

[9] S. Provoost, H. M. Lau, J. Ruwaard, and H. Riper, "Embodied conversational agents in clinical psychology: A scoping review," Journal of Medical Internet Research, vol. 19, no. 5. Journal of Medical Internet Research, p. e151, 01-May-2017.

[10] A. N. Vaidyam, H. Wisniewski, J. D. Halamka, M. S. Kashavan, and J. B. Torous, "Chatbots and Conversational Agents in Mental Health: A Review of the Psychiatric Landscape," Canadian Journal of Psychiatry, vol. 64, no. 7. SAGE Publications Inc., pp. 456-464, 01-Jul-2019.

[11] N. Novielli, I. Mazzotta, B. De Carolis, and S. Pizzutilo, "Analysing user's reactions in advice-giving dialogues with a socially intelligent ECA," Cogn. Process., vol. 13, no. SUPPL. 2, pp. 487-97, Oct. 2012.

[12] E. Hudlicka, "Virtual training and coaching of health behavior: Example from mindfulness meditation training," Patient Educ. Couns., vol. 92, no. 2, pp. 160-166, Aug. 2013.

[13] C. J. Stevens et al., "Mimicry and expressiveness of an ECA in human-agent interaction: familiarity breeds content!," Comput. Cogn. Sci., vol. 2, no. 1, p. 1, Dec. 2016.

[14] J. A. García-Casal, A. Loizeau, E. Csipke, M. Franco-Martín, M. V. Perea-Bartolomé, and M. Orrell, "Computer-based cognitive interventions for people living with dementia: a systematic literature review and meta-analysis," Aging and Mental Health, vol. 21, no. 5. Routledge, pp. 454-467, 04-May-2017. 
[15] M. Turunen et al., "Computer-based cognitive training for older adults: Determinants of adherence," PLoS One, vol. 14, no. 7, 2019.

[16] R. Jacobsen, J.-H., Stelzer, J., Fritz, T. H., Chételat, G., La Joie, R., \& Turner, "Why Musical Memory Can Be Preserved in advanced Alzeheimer's disease," Brain Sci., no. July, pp. 2438-2450, 2015.

[17] K. Fleming, S. H. Kim, M. Doo, G. Maguire, and S. G. Potkin, "Memory for emotional stimuli in patients with Alzheimer's disease.," Am. J. Alzheimers. Dis. Other Demen., vol. 18, no. 6 , pp. 340-2, Nov. 2003.

[18] S. B. Hamann, E. S. Monarch, and F. C. Goldstein, "Memory enhancement for emotional stimuli is impaired in early Alzheimer's disease," Neuropsychology, vol. 14, no. 1, pp. 8292, 2000.

[19] G. Tur and R. De Mori, Spoken language understanding: systems for extracting semantic information from speech. Wiley, 2011.

[20] J. R. Bellegarda, "Spoken Language Understanding for Natural Interaction: The Siri Experience," in Natural Interaction with Robots, Knowbots and Smartphones, Springer New York, 2014, pp. 3-14.

[21] F. Serón and C. Bobed, "VOX System: A Semantic Embodied Conversational Agent exploiting Linked Data," Multimed. Tools Appl., vol. 75, pp. 381-404, 2016.

[22] P. Cimiano and S. Kopp, "Accessing the Web of Data through embodied virtual characters," Semant. Web, vol. 1, no. 1-2, pp. 83-88, 2010.

[23] U. Waltinger, A. Breuing, and I. Wachsmuth, "Interfacing Virtual Agents with Collaborative Knowledge: Open Domain Question Answering Using Wikipedia-Based Topic Models," in Proceedings of the Twenty-Second International Joint Conference on Artificial Intelligence - Volume Volume Three, 2011, pp. 1896-1902.

[24] G. W. I. Lcock and G. W. I. Lcock, "WikiTalk: A Spoken Wikipedia-based Open-Domain Knowledge Access System."

[25] A. Breuing, "Improving human-agent conversations by accessing contextual knowledge from Wikipedia," in Proceedings - 2010 IEEE/WIC/ACM International Conference on Web Intelligence and Intelligent Agent Technology Workshops, WI-IAT 2010, 2010, pp. 428-431.

[26] T. R. Gruber, "A translation approach to portable ontology specifications," Knowl. Acquis., vol. 5, no. 2, pp. 199-220, Jun 1993.

[27] T. R. Gruber, "Toward principles for the design of ontologies used for knowledge sharing," Int. J. Hum. - Comput. Stud., vol. 43, no. 5-6, pp. 907-928, 1995.

[28] J. Cassell, "Human Representation and Intelligence in Face-to-Face Conversation,” pp. 67-84, 2001.

[29] L. Mignonneau and C. Sommerer, "Designing emotional, metaphoric, natural and intuitive interfaces for interactive art, edutainment and mobile communications," Comput. Graph., vol. 29, no. 6, pp. 837-851, Dec. 2005.

[30] J. Ortiz, A.; Aizpurua, I.; Posada, "Some techniques for avatar support of digital sto- rytelling systems," in Technologies for Interactive Digital Storytelling and Entertainment, 2003.
[31] A. García and C. Lamsfus, "AN eLEARNING PLATFORM TO SUPPORT VOCATIONAL TRAINING CENTERS ON DIGITAL SECURITY TRAINING WITH VIRTUAL TUTORS AND GRAPHICAL SPATIAL METAPHORES," Technology, pp. 1-2.

[32] A. C. Graesser, P. Chipman, B. C. Haynes, and A. Olney, "Auto tutor: An intelligent tutoring system with mixed-initiative dialogue," IEEE Trans. Educ., vol. 48, no. 4, pp. 612-618, Nov. 2005.

[33] J. C. Lester, J. C. Lester, S. G. Towns, and P. J. FitzGerald, "Achieving affective impact: Visual emotive communication in lifelike pedagogical agents,” Int. J. Artif. Intell. Educ., vol. 10, pp. 278--291, 1999.

[34] T. Rieger, “Avatar Gestures,” J. WSCG, vol. Vol. 1, pp. 379386, 2003.

[35] S. C. Marsella, W. L. Johnson, and C. LaBore, "Interactive pedagogical drama," in Proceedings of the International Conference on Autonomous Agents, 2000, pp. 301-308.

[36] D. C. Berry, L. T. Butler, and F. De Rosis, "Evaluating a realistic agent in an advice-giving task," Int. J. Hum. Comput. Stud., vol. 63, no. 3, pp. 304-327, 2005.

[37] X. Yuan and Y. S. Chee, "Design and evaluation of Elva: an embodied tour guide in an interactive virtual art gallery," Comput. Animat. Virtual Worlds, vol. 16, no. 2, pp. 109-119, 2005.

[38] E. Isaac, A; Summers, "SKOS Simple Knowledge Organization System Primer," W3C Working Group Note, 2009. [Online]. Available: https://www.w3.org/TR/skos-primer/. [Accessed: 19-Jan-2020].

[39] Amazon, "AWS Elastic compute cloud (EC2) de capacidad modificable en la nube," Amazon Web Services, Inc., 2015. [Online]. Available: //aws.amazon.com/es/ec2/. [Accessed: 19-Jan-2020].

[40] M. Jovanovik, D. Trajanov, and M. Kostovski, "Open Data Portal based on Semantic Web Technologies LDA: Linked Data Authorization View project HOBBIT: Holistic Benchmarking of Big Linked Data View project Open Data Portal based on Semantic Web Technologies," 2012.

[41] S. S. Naveen Malviya, Nishchol Mishra, N. Malviya, N. Mishra, and S. Sahu, "Developing University Ontology using protégé OWL Tool: Process and Reasoning," Int. J. Sci. Eng. Res. Vol. 2, Issue 9, vol. 2, no. 9, pp. 1-8, 2011.

[42] C. Bobed, G. Esteban, and E. Mena, "Enabling keyword search on linked data repositories: An ontology-based approach," Int. J. Knowledge-Based Intell. Eng. Syst., vol. 17, no. 1, pp. 67-77, 2013.

[43] A. Quint and P. Liu, "Scalable vector graphics," IEEE Multimed., vol. 10, no. 3, pp. 99-102, Jul. 2003.

[44] J.- Ferraiolo, "Scalable Vector Graphics (SVG) 1.0 Specification," W3C Working Group Note, 2001. [Online]. Available: https://www.w3.org/TR/2001/REC-SVG-20010904/. [Accessed: 19-Jan-2020].

[45] M. Pilgrim, HTML5: Up and Running, 1st ed. O’Reilly Media, Inc., 2010. 
[46] G. Anthes, "HTML5 Leads a Web Revolution," Commun. ACM, vol. 55, no. 7, pp. 16-17, Jul. 2012.

[47] L. Stevens, R. Owen, L. Stevens, and R. Owen, "The Truth About SVG: The Flash Challenger That Was, Wasn't, and Now...," in The Truth About HTML5, Apress, 2014, pp. 135151 .

[48] K. Man \`ena, V.; S $`^{`}$ pilka, R.; Rybensk $\backslash$ 'a, “A comparison of free and open source technologies suitable for future authoring tools in education," in Future Communication Technology and Engineering: Proceedings of the 2014 International Conference on Future Communication Technology and Engineering (FCTE 2014) - CRC Press Book, pp. 83-86.
[49] Gates, N. J., Rutjes, A. W., Di Nisio, M., Karim, S., Chong, L. Y., March, E., Martínez, G., Vernooij, R. W., 2019. Computerised cognitive training for maintaining cognitive function in cognitively healthy people in late life. The Cochrane database of systematic reviews 3, CD012277. URL: http://www.ncbi.nlm.nih.gov/pubmed/30864187http://www.pu bmedcentral.nih.gov/articlerender.fcgi?artid=PMC6414816, doi:10.1002/14651858.CD012277.pub2.

[50] McBride, B., 2001. Jena: Implementing the rdf model and syntax specification, in: Proceedings of the Second International Conference on Semantic Web - Volume 40, CEUR-WS.org, Aachen, DEU. p. 23-28. 